\title{
Alemtuzumab induction in pediatric kidney transplantation
}

\author{
Kaabak MM, Babenko NN, Samsonov DV, Sandrikov VA, Maschan \\ AA, Zokoev AK. Alemtuzumab induction in pediatric kidney \\ transplantation.
}

Abstract: Recipient parenchymal lymphatic cells are crucial for direct and indirect pathways of allorecognition. We proposed that alemtuzumab, being infused several weeks pretransplant could eradicate peripheral lymphatic cells and promote donor-specific tolerance. We present here a single center, retrospective review of 101 consecutive living-donor kidney transplantations to pediatric patients aged from seven month to $18 \mathrm{yr}$, performed between September 2006 and April 2010. Immunosupression protocol included two $30 \mathrm{mg}$ doses of alemtuzumab: first given 12-29 d prior to transplantation and second at the time of transplantation. Maintenance immunosupression was based on combination of low dose and wide range CNI and mycophenolate. Patients were followed for $3.8 \pm 1.4 \mathrm{yr}$ and protocol biopsies were taken one month, one, and three yr post transplant. The Kaplan-Meier graft and patient survival was $96 \%$ and $97 \%$ for one yr, $89 \%$ and $93 \%$ for three yr. Biopsy proven acute rejection developed in $26 \%$ patients at one yr and in $35 \%$ at two yr, no rejections occurred beyond two yr. We conclude that alemtuzumab pretreatment prior to living related donor kidney transplantation allows to reach satisfactory middle-term results in pediatric patients with wide range and low CNI concentrations.

\section{Michael M. Kaabak ${ }^{1}$, Nadezda N. Babenko², Dmitry V. Samsonov ${ }^{3}$, Valery A. Sandrikov ${ }^{4}$, Alexey A. Maschan $^{5}$ and Alan K. Zokoev ${ }^{2}$}

${ }^{1}$ Organ Transplant Division, ${ }^{2}$ Kidney Transplant Department, Russian Scientific Center of Surgery, Moscow, Russia, ${ }^{3}$ New York Medical College, Valhalla, NY, USA, ${ }^{4}$ Diagnostic Division, Russian Scientific Center for Surgery, ${ }^{5}$ Federal Clinical Research Center for Pediatric Hematology, Oncology and Immunology, Moscow, Russia

Key words: alemtuzumab - pediatric kidney transplantation - induction therapy - steroid free immunosupression

Michael Kaabak, Department of Kidney

Transplantation, National Research Center for

Surgery, Abrikosovsky lane 2, Moscow 119992, Russia

Tel.: +74 992481344

Fax: +74 997664685

E-mail: kaabak@hotmail.com

Accepted for publication 19 December 2012
Alemtuzumab (Campath-1H, MabCampath) is a humanized IgG1 monoclonal antibody directed against CD52, a glycoprotein expressed on mononuclear cells, including $\mathrm{T}$ and $\mathrm{B}$ lymphocytes, monocytes, and natural killer cells $(1,2)$. Alemtuzumab is the most powerful of the currently used lymphocyte-depleting agents; it brings about a rapid and sustained depletion of circulating and peripheral lymphocytes $(3,4)$. Maximal depletion of peripheral lymphocytes takes between two and $10 \mathrm{~d}$ and has been

\footnotetext{
Abbreviations: ABOi, ABO-incompatible; AUC, area under the curve; BKV, BK virus; CMV, cytomegalovirus; $\mathrm{CNI}$, calcineurin inhibitor; CsA, cyclosporine A; EBV, Epstein-Barr virus; ESRD, end-stage renal disease; FSGS, focal segmental glomerulosclerosis; GFR, glomerular filtration rate; HLA, human leukocyte antigen; HUS, hemolytic uremic syndrome; IFTA, interstitial fibrosis and tubular atrophy; MMF, mycophenolate mofetil; NATs, nucleic acid test; PCP, pneumocystis carinii pneumonia; PCR, polymerase chain reaction; PSI, proliferative signal inhibitor; PTLD, post-transplant lymphoproliferative disorder.
}

confirmed in both nonhuman primates and transplant patients $(3,5)$.

Alemtuzumab has been used as an induction agent in renal transplantation since the first (1998) report by Calne et al. (6), who demonstrated that the use of alemtuzumab induction allowed transplant recipients to be maintained on a low-dose cyclosporine monotherapy. Subsequent five-yr follow-up confirmed that under this immunosuppressive protocol, the patient and graft survival was similar to that achieved with conventional therapy (7). A few years later, the Pittsburgh group demonstrated promising three-yr survival rates with low-dose tacrolimus monotherapy after alemtuzumab induction (8). According to UNOS, alemtuzumab induction was utilized in $14.1 \%$ of all kidney transplantations performed in the United States between 2000 and 2010 (based on OPTN data as of January 14, 2011). In a recently published prospective randomized trial (9), alemtuzumab was associated with lower rates of acute rejection than basiliximab in low immunological risk patients and was 
associated with similar efficacy as compared with rabbit anti-thymocyte globulin in high-risk patients. The superiority of alemtuzumab over daclizumab was also demonstrated in a randomized trial (10). Calne and Watson's review (11) suggested that alemtuzumab induction reduced the dosage required for maintenance immunosuppression; there was an increased proportion of regulatory $\mathrm{T}$ cells after alemtuzumab use.

The use of alemtuzumab in pediatric kidney transplantation is relatively limited. The first report of four patients was unfavorable: rejection was seen in three of four patients, including two antibody-mediated rejections (12). The largest series of pediatric patients was published by the Pittsburgh group, whose protocol included pretreatment of recipients with a single dose of alemtuzumab as well as tacrolimus monotherapy (13). An average four-yr follow-up of 42 pediatric patients showed promising results in terms of safety, efficacy, and tolerability (14).

We modified the protocol utilized by the Pittsburgh group. Our patients received two doses of alemtuzumab, pretreatment with alemtuzumab two to three wk before the transplantation and the second alemtuzumab dose on the day of transplantation. The rationale for this specific protocol is an attempt to achieve maximal peripheral lymphocyte depletion during and after the transplantation. The depletion of recipient and donor antigen-presenting cells is expected to induce the abrogation of direct and indirect allorecognition and to impair costimulatory signaling $(15,16)$.

This study evaluates the advantages and disadvantages of this strategy with an emphasis on the analysis of recipient survival, graft loss, acute rejection, and infections.

\section{Materials and methods}

This single-center, retrospective review covered alemtuzumab induction therapy for 101 consecutive living donor kidney transplantations in pediatric patients between seven months and $18 \mathrm{yr}$ of age, performed between September 2006 and April 2010 at the Russian Scientific Center of Surgery, Moscow, Russia. The alemtuzumab induction protocol was reviewed and approved by our institution's Ethics Committee, and informed consent was received from the patients' parents or guardians. Our institution used a twodose alemtuzumab induction regimen: one dose of $30 \mathrm{mg}$ 12-29 d prior to the transplantation $(18.0 \pm 3.1)$ and one dose at the time of transplantation, $30 \mathrm{mg}$ for children over $10 \mathrm{~kg}$ and $15 \mathrm{mg}$ for children weighing $10 \mathrm{~kg}$ or less. The patients were followed for $3.8 \pm 1.4 \mathrm{yr}$. Two patients were unavailable for follow-up because of a change of residence 213 and 912 d post-transplant.

All patients were initially treated with a combination of a CNI, MMF, and steroids. The choice of CNI (cyclosporine or tacrolimus) was initially dependent on drug availability; beginning in April 2009, we began a randomized trial of cyclosporine or tacrolimus (clinicaltrials.gov identifier: NCT01346397). The trough levels of tacrolimus were adjusted to $8-12 \mathrm{ng} / \mathrm{mL}$ for the first $10 \mathrm{~d}$ and $2-8 \mathrm{ng} / \mathrm{mL}$ starting from day 11 . The levels of cyclosporine were measured before dose intake, at one and three $\mathrm{h}$ after administration of the dose and adjusted for the target AUC: $3500-4500 \mathrm{ng} / \mathrm{mL} / \mathrm{h}$ and $\mathrm{C} 0 \quad 100-200 \mathrm{ng} / \mathrm{mL}$ until day 10 ; AUC around 2500 and $\mathrm{C} 0$ 75-150 until day 30; AUC 1500-2000 and C0 50-100 thereafter. AUC was calculated using a Gaspary equation (17). All patients received $10 \mathrm{mg} / \mathrm{kg}$ of methylprednisolone intravenously before reperfusion. From day one, all patients received $60 \mathrm{mg} / \mathrm{m}^{2}$ of prednisone (maximal dose $80 \mathrm{mg}$ ). Steroids were discontinued in patients with good early graft function when the desired level of CNI was reached, usually by day five after transplantation. In patients with impaired graft function, steroids were withdrawn if weekly graft biopsies did not show any signs of rejection. MMF was introduced at a dose of $1200 \mathrm{mg} / \mathrm{m}^{2} / \mathrm{d}$ after the resolution of neutropenia caused by the induction therapy (white blood cell count above $3.0 \times 10^{6} / \mathrm{mL}$ ). MMF was discontinued and replaced by azathioprine in one patient because of intolerable side effects (diarrhea). In cases when reduction in immunosuppression was clinically indicated because of acute CMV or BK infection or rising EBV-PCR titers, the first choice was the initial reduction in the CNI dosage by $50 \%$. If further reduction was necessary and proteinuria did not exceed $1 \mathrm{~g} /$ $\mathrm{d}$, the CNI was replaced with a PSI. Both everolimus and sirolimus were used; the choice was dependent on drug availability, with the target trough blood level at $2-8 \mathrm{ng} / \mathrm{mL}$ for both drugs. In case of proteinuria $>1 \mathrm{~g} / \mathrm{d}$, monotherapy with MMF was also allowed (Fig. 1).

ABOi transplant recipients received a similar induction and maintenance protocol except for pretransplant plasmapheresis, which was performed in six of eight ABOi children to achieve the target levels of isoagglutinins. The number of plasmapheresis treatments was low: $1.5 \pm 1.1$ per patient (Table 1); the volume of removed plasma during one session was as high as $150 \%$ of the total volume of circulating plasma. After the last plasmapheresis, the titer of isoagglutinins was checked twice, on two consecutive days. If the titer was low (below eight, according to the tube technique method) and was not rising, we performed transplantation. Two children had a low original isoagglutinin titer and did not need plasmapheresis.

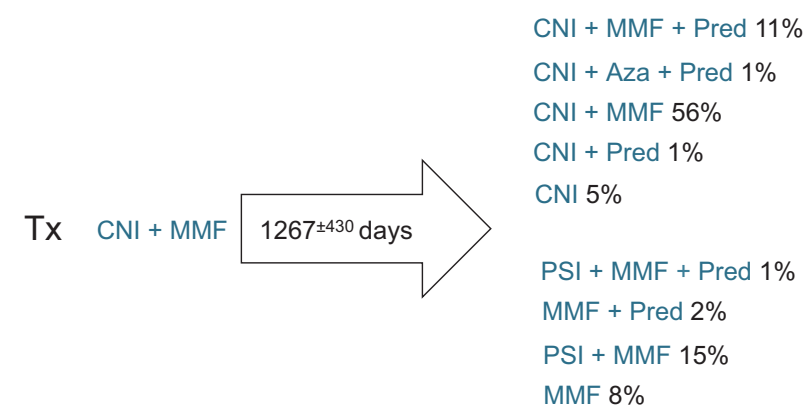

$84 \%$ steroid free, $26 \%$ CNI free

Fig. 1. Evolution of maintenance immunosuppression. 


\section{Kaabak et al.}

Protocol biopsies were performed on day 30 , one, and three yr after transplantation. Graft biopsies were performed also to clarify the reason for kidney dysfunction, which was defined as the elevation of either serum creatinine or proteinuria over $30 \%$ above baseline, on two consecutive tests. Renal allograft rejection was diagnosed by allograft biopsy. Rejection episodes were treated by a switch from CsA to Tacro and then by a patient-specific combination of either three doses of high-dose IV steroids every $48 \mathrm{~h}$ $\left(500 \mathrm{mg} / \mathrm{m}^{2}\right.$ during first month and $400 \mathrm{mg} / \mathrm{m}^{2}$ thereafter) or three doses of IV steroids followed by the addition of an oral steroid recycle starting with $25 \mathrm{mg} / \mathrm{m}^{2}$ of prednisone with a rapid taper or oral steroid re-administration. Alternatively, an oral steroid recycle was used without IV steroids, with the addition or increase in the MMF dosage.

Table 1. Donors' and recipients' demography

\begin{tabular}{lll}
\hline & $\mathrm{n}$ & $(\%)$ \\
\hline Donor & 101 & 100.0 \\
$\quad$ Female/male & $74 / 27$ & $73.3 / 26.7$ \\
$\quad 39 \pm 8.1$ & \\
\hline
\end{tabular}

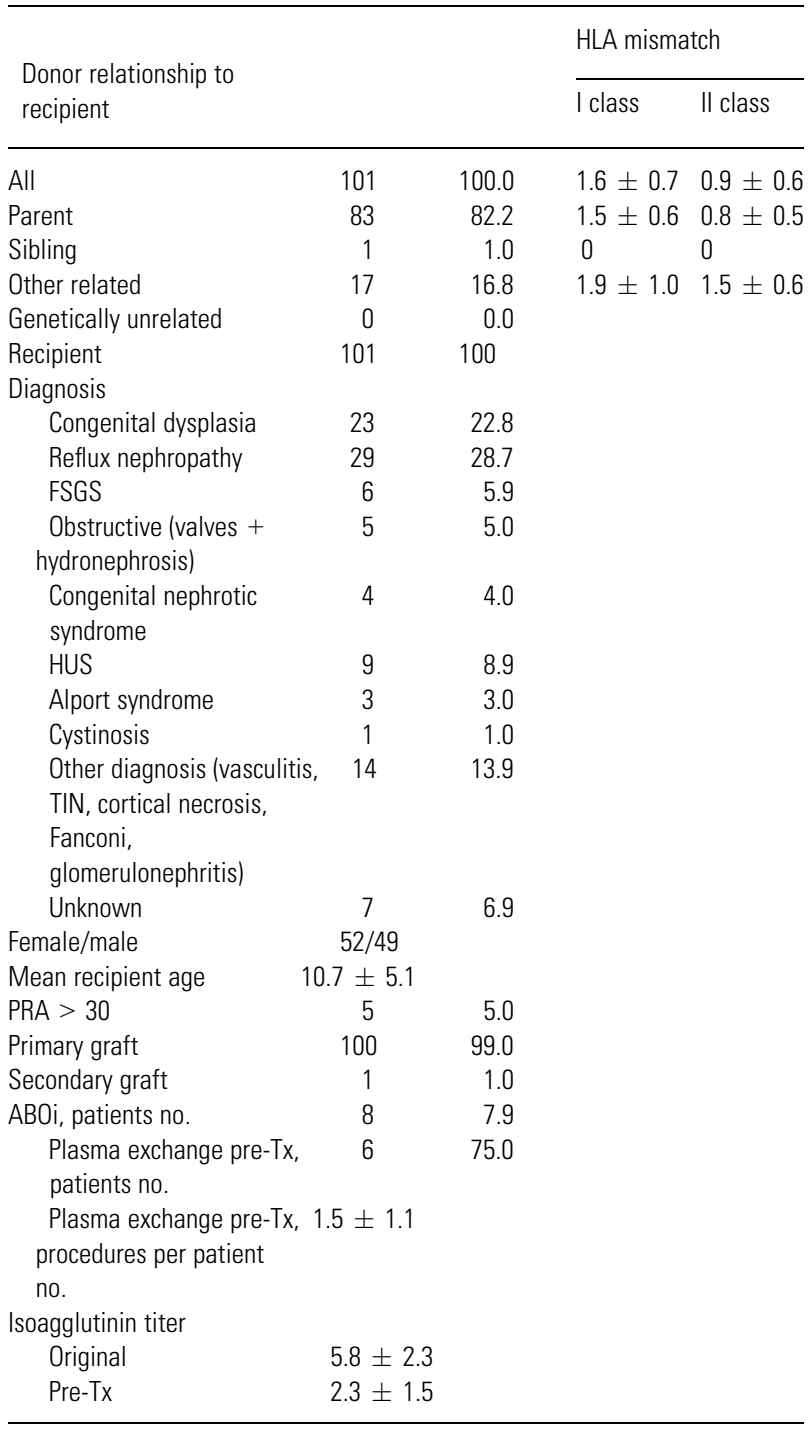

PRA, panel reactive antibodies.
The severity and acuteness of the rejection, anticipated side effects of medications, and available diagnostic and treatment options determined the design of a specific antirejection treatment for each individual patient.

Viral infections, CMV, BKV, and EBV were defined as the presence of viremia (for BKV, also viruria) by DNA capture or more recently by quantitative PCR analysis.

All patients received prophylaxis against $\mathrm{PCP}$ with daily $\mathrm{TMP} /$ sulfa for six months. Viral prophylaxis with valganciclovir was given to all patients regardless of their pretransplant CMV and EBV status for three wk after transplantation, with subsequent CMV-PCR monitoring every month for the first year and every three months thereafter. In the event of detectable CMV viremia, preemptive therapy with valganciclovir was re-introduced.

Patient and graft survival, the incidence of rejection, and the incidence of viral infections were analyzed for the whole group as well as for the cyclosporine and tacrolimus groups.

\section{Results}

Patient characteristics and survival

The recipient and donor demography is summarized in Table 1, along with the HLA mismatches in each category. The major causes of ESRD were reflux nephropathy in $28.7 \%$ and congenital dysplasia in $22.8 \%$. Diseases that can recur in the transplant were found in $5.9 \%$ with FSGS, $8.9 \%$ with HUS. The average HLA mismatch was $2.5 \pm 1.0$.

Eight recipients received an ABOi kidney, A to $0(n=3)$ and $B$ to $0(n=3)$, A to $B(n=1)$ and $\mathrm{B}$ to $\mathrm{A}(\mathrm{n}=1)$; their characteristics are summarized in Table 1.

The Kaplan-Meier patient actuarial survival at one, two, and three yr was $97.0 \pm 2.0 \%$, $94.0 \pm 3.5 \%$, and $93.0 \pm 2.1 \%$. There were eight $(7.9 \%)$ recipient deaths with a functioning graft (Table 2 Part a).

\section{Graft survival and function}

The graft survival at one, two, and three yr was $96.0 \pm 2.0 \%, 92.1 \pm 4.0 \%$, and $89.1 \pm 3.6 \%$. The mean serum creatinine $(\mathrm{mg} / \mathrm{dL})$ was $1.0 \pm 0.5,1.1 \pm 0.7,1.2 \pm 0.8$, and the GFR (measured by the classic Schwartz formula) was $91.6 \pm 33.7,84.0 \pm 37.2$, and $82.8 \pm 30.5 \mathrm{~mL} / \mathrm{min}$ at one, two, and three yr, respectively. Proteinuria $(\mathrm{mg} / 24 \mathrm{~h})$ was $271 \pm 315, \quad 526 \pm 586$, and $180 \pm 301$ at one, two, and three yr. Immediate function was observed in $93 \%$ of allografts. Six children required one session of hemodialysis during the first postoperative week, and one child received dialysis for four wk until graft function recovered. The loss of five renal allografts was related to graft artery thrombosis in one patient on day 0 , noncompliance in three adolescent patients on days 433, 836, and 1018 post-trans- 
Table 2. Mortality and particular postoperative courses

\begin{tabular}{|c|c|c|c|c|c|c|}
\hline No. & \multicolumn{2}{|c|}{ Gender } & Age at tx & Postoperative day & \multicolumn{2}{|l|}{ Cause of death } \\
\hline \multicolumn{7}{|c|}{ (a) Causes of death in 8 of 101 alemtuzumab-treated patients } \\
\hline 1 & \multicolumn{2}{|l|}{ Girl } & $7 \mathrm{yr}$ & 452 & \multicolumn{2}{|l|}{ Respiratory failure due to ascending paralysis } \\
\hline 2 & \multicolumn{2}{|l|}{ Boy } & 7 months & 165 & \multicolumn{2}{|c|}{$\begin{array}{l}\text { Brain edema due to electrolyte disorders caused by acute kidney obstruction with subsequent urine outflow } \\
\text { restoration }\end{array}$} \\
\hline 3 & \multicolumn{2}{|l|}{ Girl } & $8 \mathrm{yr}$ & 1457 & \multicolumn{2}{|c|}{ Meningococcal meningoencephalitis } \\
\hline 4 & \multicolumn{2}{|l|}{ Girl } & $3 \mathrm{yr}$ & 420 & \multicolumn{2}{|l|}{ Unknown } \\
\hline 5 & \multicolumn{2}{|l|}{ Boy } & $2 \mathrm{yr}$ & 548 & \multicolumn{2}{|l|}{ PCP } \\
\hline 6 & \multicolumn{2}{|l|}{ Girl } & 11 months & 26 & \multicolumn{2}{|l|}{ Pneumonitis, pathogen not specified } \\
\hline 7 & \multicolumn{2}{|l|}{ Girl } & $7 \mathrm{yr}$ & 891 & \multicolumn{2}{|l|}{ Pneumonitis, pathogen not specified } \\
\hline 8 & \multicolumn{2}{|l|}{ Boy } & $15 \mathrm{yr}$ & 273 & \multicolumn{2}{|l|}{ Pneumonitis, pathogen not specified } \\
\hline \multicolumn{6}{|c|}{ (b) Clinically apparent EBV and CMV infections and PCP } & Outcome \\
\hline 1 & Boy & $4 \mathrm{yr}$ & \multicolumn{3}{|c|}{$\begin{array}{l}\text { EBV-associated flu like syndrome } 6 \text { months post-transplant, resolved after switch from Tacro + MMF to } \\
\text { Rapamune }\end{array}$} & 4 yr, excellent graft function \\
\hline 2 & Boy & $2 \mathrm{yr}$ & \multicolumn{3}{|c|}{ PCP developed 1 month post-transplant, completely resolved on trimethoprim-sulfametoxasole } & $\begin{array}{l}3 \mathrm{yr} \text {, impaired graft function (GFR } \\
20 \mathrm{~mL} / \mathrm{min} \text { ) }\end{array}$ \\
\hline 3 & Boy & $6 \mathrm{yr}$ & \multicolumn{3}{|c|}{$\begin{array}{l}\text { CMV-associated pneumonia on day 230, } 4 \text { wk apart of steroid-resistant rejection treatment with Campath, } \\
\text { completely resolved with 4-wk valganciclovir }\end{array}$} & 3 yr, excellent graft function \\
\hline 4 & Boy & $4 \mathrm{yr}$ & \multicolumn{3}{|c|}{ CMV-associated enteritis 1 yr post-transplant, resolved with 2-wk valganciclovir } & 3 yr, excellent graft function \\
\hline
\end{tabular}

(c) Reasons for not withdrawal the steroids in 5 of 101 alemtuzumab-treated patients

1 Boy 7 months Gastrointestinal infection on day 7 with acute graft failure, CNI was suspended for 4 days, graft function recovered promptly, CNI target level was achieved slowly and proper time for steroid withdrawal was missed

2 Girl $8 \mathrm{yr} \quad$ Delayed graft function until day 28, we preferred to left her on steroids then undergo every week biopsy

3 Boy $5 \mathrm{yr} \quad$ Decortication due to profound and prolonged hypoglycemia, PSI was used instead CNI to avoid neurotoxicity

4 Girl $10 \mathrm{yr} \quad$ Slow graft function (normal creatinine on day 21) caused by artery thrombosis with restoration of blood flow within $1 \mathrm{~h}$

5 Girl $13 \mathrm{yr} \quad$ Slow graft function (normal creatinine on day 44) caused by venous kinking with restoration of blood outflow

Vulnerability to rejection due to ischemic injury made us continue within $4 \mathrm{~h}$ steroids in these two children

plant and to a refractory rejection in one patient on day 1409.

Mean follow-up of the ABOi recipients over $3.3 \pm 0.6 \mathrm{yr}$ showed no graft loss or patient death. One child has significantly impaired graft function because of a refractory rejection, with a serum creatinine of $6.4 \mathrm{mg} \%$ and a GFR of $11 \mathrm{~mL} / \mathrm{min}$ at $2.5 \mathrm{yr}$ post-transplant. She is being reevaluated for retransplantation. The other seven ABOi recipients have good renal function. The mean serum creatinine $(\mathrm{mg} / \mathrm{dL})$ at last follow-up was $1.1 \pm 0.3$, with a GFR of $70.7 \pm 21.6 \mathrm{~mL} / \mathrm{min}$, and no significant proteinuria $(130 \pm 112 \mathrm{mg} / 24 \mathrm{~h})$.

\section{Immunosuppressive therapy}

All patients received intravenously of methylprednisolone $10 \mathrm{mg} / \mathrm{kg}$ before reperfusion. From day one, all patients received $60 \mathrm{mg} / \mathrm{m}^{2}$ of prednisone (maximal dose was $80 \mathrm{mg}$ ). Steroids were discontinued after $7.0 \pm 6.4 \mathrm{~d}$ in 96 of 101 patients with good primary graft function after achieving the target levels of the CNI. Reasons for steroid continuation are indicated in Table 2 Part b.
CNIs (cyclosporine in 63 patients and tacrolimus in 36 patients) were started on day zero $(0.0 \pm 0.3)$. Two patients did not receive a CNI (one patient was started on sirolimus because of severe central nervous system dysfunction and was switched to cyclosporine at day 50; another patient received an HLA identical kidney from his brother).

In the cyclosporine group (63 patients at day one), $19(30 \%)$ patients were subsequently switched to tacrolimus $375 \pm 281 \mathrm{~d}$ posttransplant because of rejection. Three yr posttransplantation, $15(24 \%)$ and $48(76 \%)$ children were receiving CNI-free and steroid-free immunosuppression, respectively. In the tacrolimus group three yr post-transplantation, eight $(22 \%)$ and $33(92 \%)$ children were receiving CNI-free and steroid-free immunosuppression, respectively. Indications for discontinuation of the CNI were clinical evidence of overimmunosuppression, including recurrent viral infections, either clinical or laboratory: positive NATs for EBV, CMV, BKV in blood, high levels $\left(>10^{7}\right)$ of $\mathrm{BKV}$ in urine, and progressive morphological signs of nephrotoxicity. 


\section{Kaabak et al.}

\section{Rejection}

The incidence of acute rejection is shown in Fig. 2. Any tubulitis, including subclinical changes, was diagnosed as acute rejection (subclinical seven of $37 \%$ or $19 \%$ of all rejections). The incidence of cumulative biopsy-proven rejection at one, two, and three yr was $26 \%$, $35 \%$, and $35 \%$, respectively. The degree of rejections according to Banff 97 score was as follows: among 37 rejections, $14(38 \%)$ were borderline; $19(51 \%), 1 \mathrm{a} ; 1(3 \%), 1 \mathrm{~b}$; and $3(8 \%), 2 \mathrm{a}$. Among 49 patients operated between August 2006 and April 2008, acute rejection developed in 29\% and $39 \%$ for one and two yr, respectively, while among 52 patients operated between May 2008 and April 2010, the figure was $23 \%$ and $31 \%$, respectively (Fig. 2b). We attributed the decreas- ing incidence to the accumulation of experience with alemtuzumab and more timely MMF administration; fear of overimmunosuppression during the first few months may also have played a role.

Treatment of acute rejection: 25 of 37 acute rejection episodes were treated with steroids (intravenously and/or orally). The remaining 12 patients were treated only with increased oral immunosuppression (switching from cyclosporine to tacrolimus or increasing the MMF dose). Two patients had steroid-resistant rejections and were successfully treated with alemtuzumab on days 206 and 383. In four patients, rejections were irreversible (three noncompliant patients) and led to graft loss on days 433, 836, 1018, and 1409.

Mean Banff scores for IFTA, arteriolar hyalinosis, chronic fibrointimal thickening, and
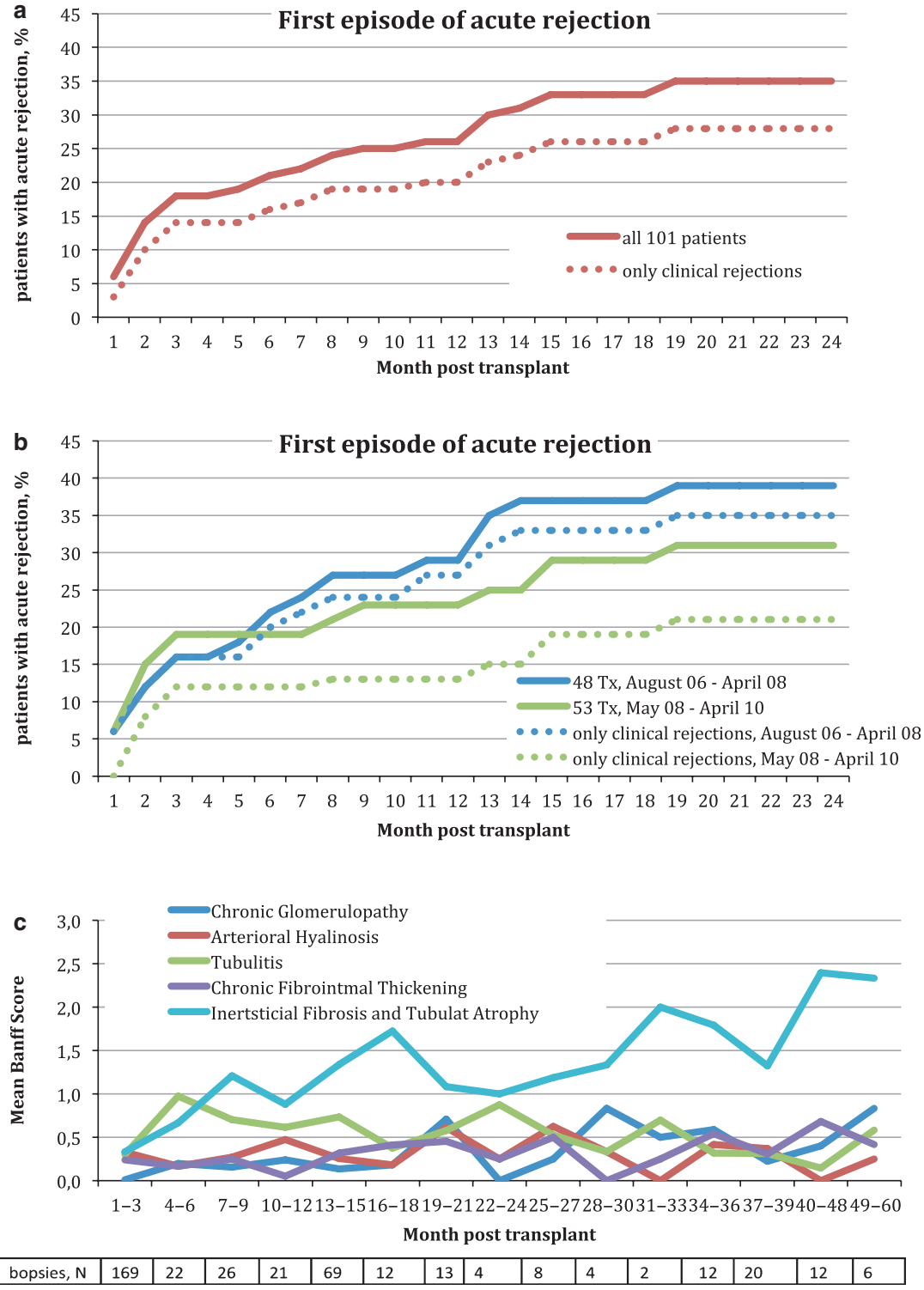

Fig. 2. (a) Acute rejection in 101 alemtuzumab treated patients; (b) patients stratified by time of transplantation. Incidence of clinical rejection depicted by dotted lines. (c) Mean Banff scores for chronic interstitial fibrosis, tubular atrophy, arteriolar hyalinosis, chronic fibrointimal thickening, and chronic glomerulopathy. 
chronic glomerulopathy are presented in Fig. 2c. The number of long-term biopsies was not sufficient to provide a definite conclusion, but we can see a trend toward a slight increase in IFTA and a very moderate presence of other chronic changes and tubulitis over time post-transplant.

\section{Infections}

All patients received prophylaxis with valganciclovir for three wk after transplantation. CMV, EBV, and BK viremia were monitored monthly during the first post-transplant year and every three months thereafter. In cases of detectable CMV viremia, preemptive therapy with valganciclovir was re-introduced. CMV and BKV viremia were highest during the first three months and reached $30 \%$ and $25 \%$ of positive NATs. Beyond one yr, the level of CMV did not exceed $10 \%$, and BKV viremia was $<5 \%$. EBV viremia occurred with increasing frequency and reached $20 \%$ by year two. The incidence of BK viruria also increased with time, exceeding $50 \%$ by year two (Fig. 3 Part 1) and correlated well with the previously observed prevalence of BKV infection in our population (18). We did not detect any cases of PTLD; however, two children developed lymphadenopathy associated with EBV viremia that improved after discontinuation of the CNI. The incidence of EBV- and CMV-associated infections, as well as PCP, is summarized in Table 2 Part c. Immunosuppression was decreased in cases of $\mathrm{BK}$ viruria of above $10^{7}$ copies $/ \mathrm{mL}$ or repeated $\mathrm{BK}$ or $\mathrm{EBV}$ viremia as well as recurrent $\mathrm{CMV}$ viremia.

Figs. 4-6 demonstrate the evolution of viral loads depending on immunosuppression. It is evident that both tacrolimus- and cyclosporinebased immunosuppression were associated with an increased BKV infection rate, while CNI-free immunosuppression was associated with stabilization and a declining trend. The high viral infection rate at the beginning of CNI-free period is explained by a previous CNI exposure. There were no definite cases of allograft nephropathy associated with BKV.
Fig. 3. (a) Positive nucleic acid tests regardless of viral bodies concentrations. (b) BK viruria more than $10^{7}$ copies $/ \mathrm{mL}$ and CMV of $10^{3}$ or more copies $/ \mathrm{mL}$ generally accepted as "dangerous" viral loads considered as positve.
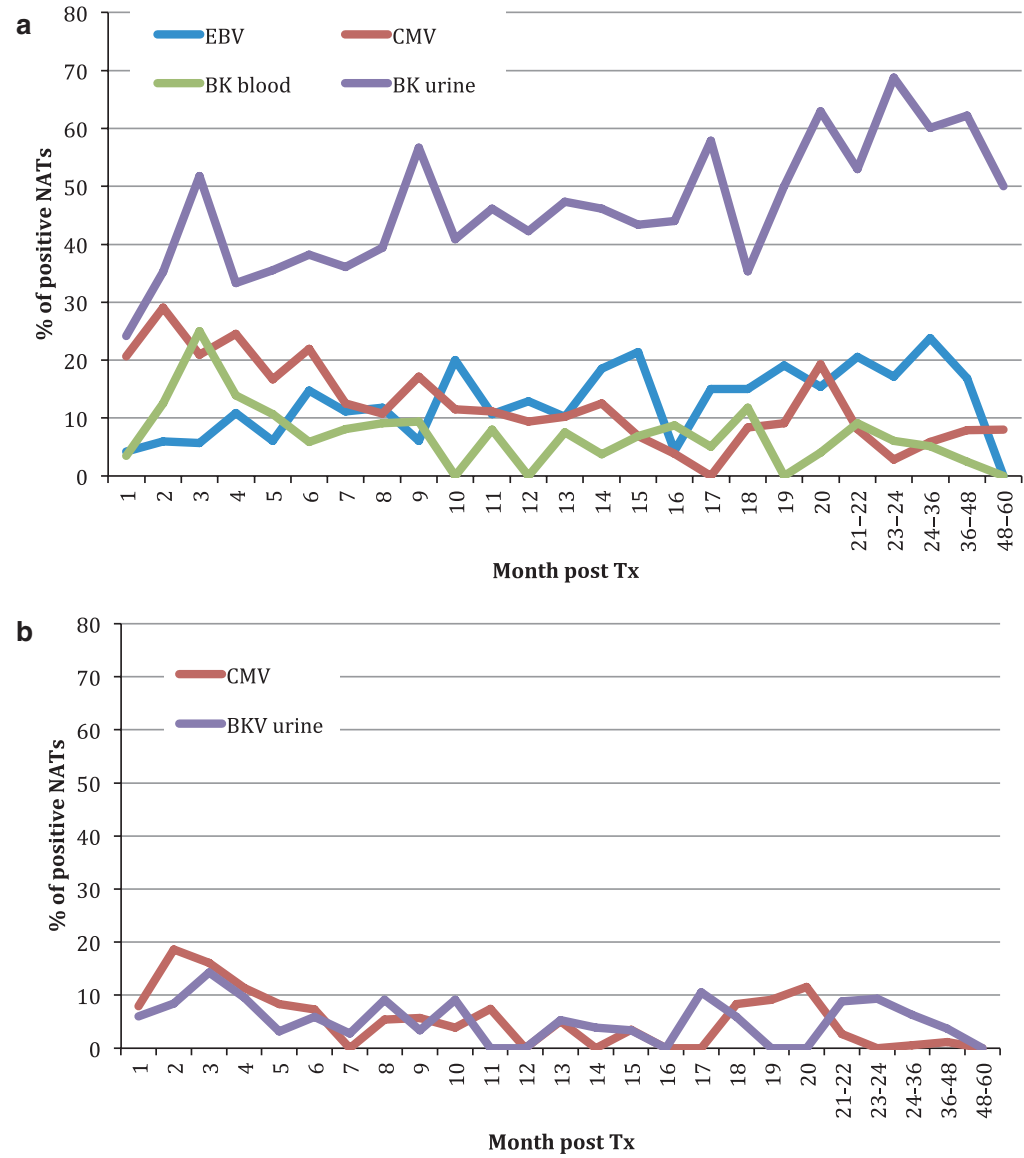

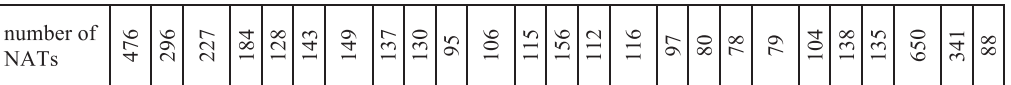


Kaabak et al.

\section{Discussion}

Antibody induction therapy is commonly utilized in renal transplant patients. In comparison with other induction therapies, alemtuzumab has been shown to be more effective in preventing acute rejection episodes (9) and allows lower doses of CNI monotherapy $(6,8)$. In our study, we implemented the Pittsburgh group strategy, which suggests that lymphoid depletion prior to graft revascularization might result in the development of partial tolerance to the allograft. We enhanced this strategy by giving the first dose of alemtuzumab two to three wk prior to living donor kidney transplantation and the second dose on the day of transplantation. The resulting peripheral lymphocyte depletion should prevent the activation of alloimmune response via direct and indirect allorecognition. Because IL-2 inhibition may interfere with tolerance induction, our second goal was to minimize CNI exposure in the post-transplant period. This theoretically justified strategy is also strengthened by the practical needs of the majority of Russian kidney recipi- ents. Most of our patients live far away from the transplant center, and the availability of local drug level monitoring is often limited. The disadvantage of this strategy is the early administration of immunosuppression, with the need to perform major surgery in immunosuppressed children. In addition, the optimal use of mycophenolate is compromised by the alemtuzumabinduced neutropenia. Mycophenolate dosage has to be increased proportionally as the bone marrow recovers, and this required prolonged and careful patient monitoring. We attribute the trend of decreased rejection rates (Fig. 2b) in more recent cases to the accumulation of experience and proper mycophenolate administration.

This study represents the largest single-center series of pediatric kidney transplant recipients receiving alemtuzumab pretreatment. The three$\mathrm{yr}$ patient and graft survival rates were $93.0 \pm 2.1 \%$ and $89.1 \pm 3.6 \%$, respectively. An historical control group of 37 live donor pediatric kidney transplantations performed at our center between November 2002 and September
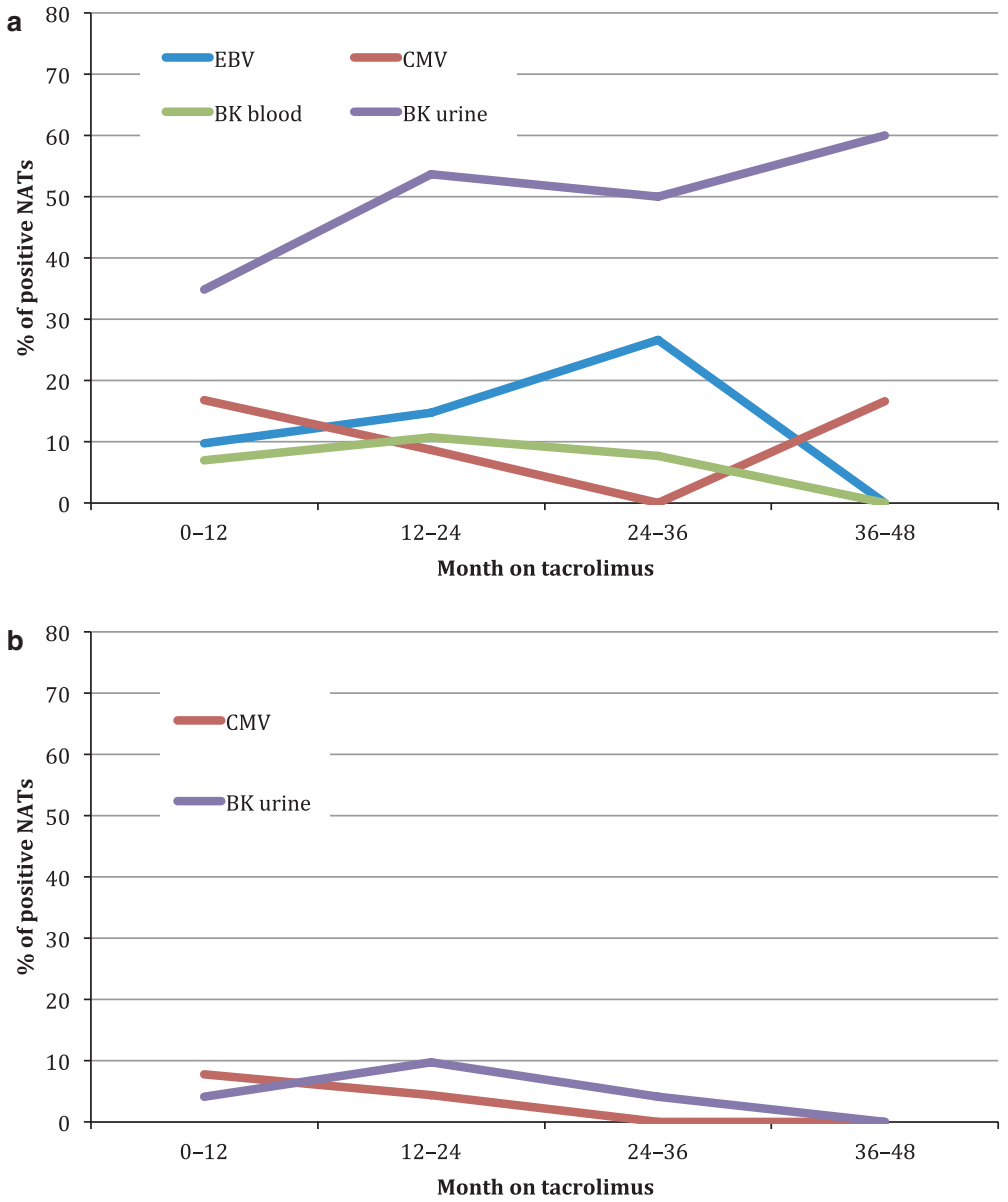

\begin{tabular}{|l|l|l|l|l|}
\hline $\begin{array}{c}\text { number } \\
\text { of NUTs }\end{array}$ & 1049 & 346 & 111 & 22 \\
\hline
\end{tabular}

Fig. 4. (a) Tacrolimus treated patients at the time of sampling. Positive nucleic acid tests regardless of viral load, as a function of time on tacrolimus. (b) Tacrolimus treated patients at the time of sampling. BK viruria more than $10^{7}$ copies $/ \mathrm{mL}$ and CMV of $10^{3}$ or more copies/ $\mathrm{mL}$ - generally accepted as "dangerous" viral loads considered as positve. 
Fig. 5. (a) Cyclosporine treated patients at the time of sampling. Positive nucleic acid tests regardless of viral load, as a function of time on cyclosporine. (b) Cyclosporine treated patients at the time of sampling. BK viruria more than $10^{7}$ copies/mL and CMV of $10^{3}$ or more copies $/ \mathrm{mL}$ - generally accepted as "dangerous" viral loads - considered as positve.
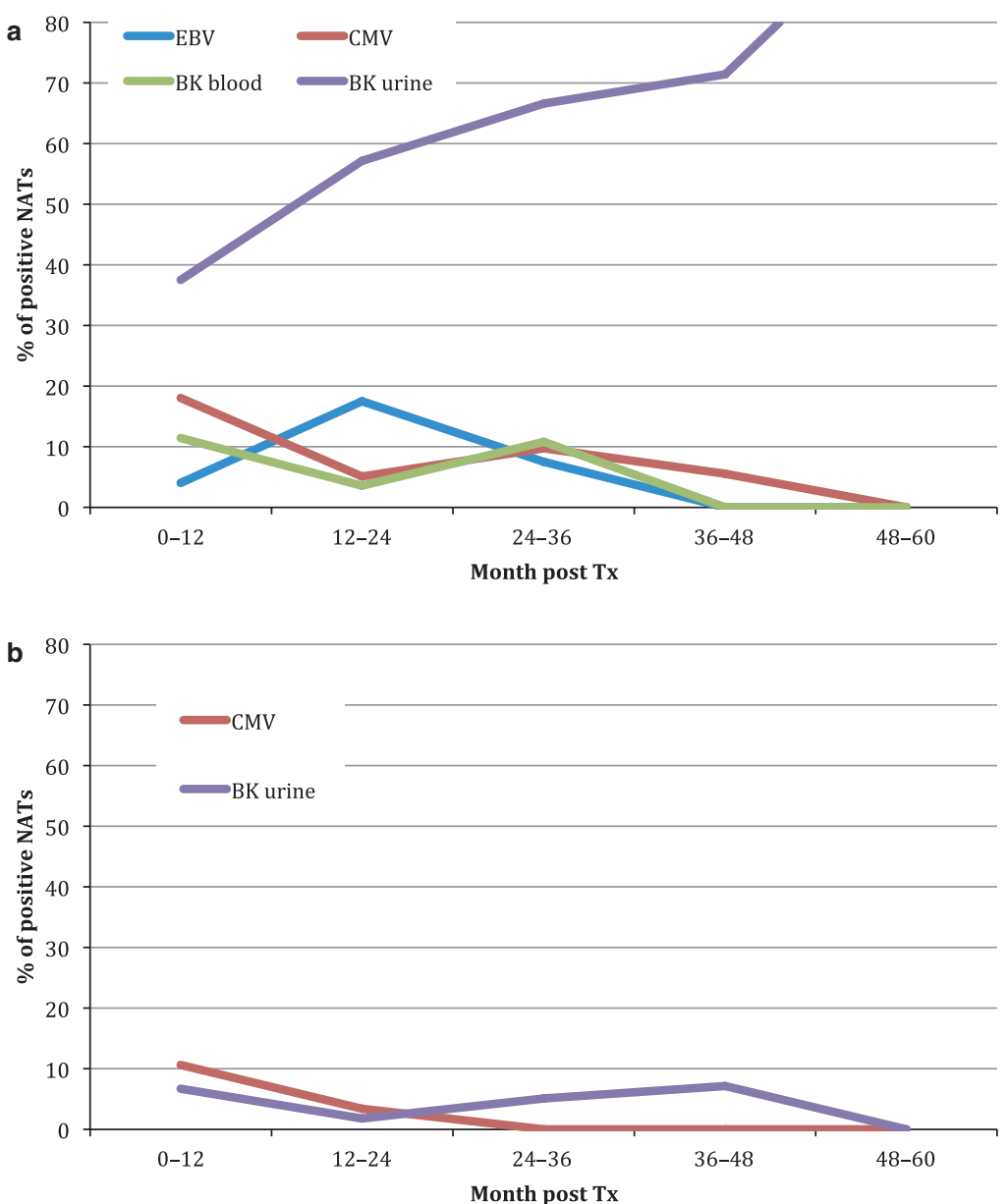

\begin{tabular}{|l|l|l|l|l|l|}
\hline $\begin{array}{l}\text { number } \\
\text { of NATs }\end{array}$ & 917 & 228 & 157 & 80 & 6 \\
\hline
\end{tabular}

2006 with Zenapax induction demonstrated a trend toward lower three-yr patient and graft survival rates: $88.9 \pm 6.1 \%$ and $81.1 \pm 6.3 \%$, correspondingly. The graft survival rate in alemtuzumab group is comparable to and patient survival is lower than living donor kidney transplant recipients reported by NAPRTCS (19), where three-yr patient and graft survival of live donor pediatric kidney transplant recipients was $97.9 \pm 0.5 \%$ and $91.3 \pm 0.6 \%$, respectively, for transplants performed between 1996 and 2010. Lower patient survival and comparable graft survival can be attributed to inadequate availability of dialysis to children in some Russian regions; in those cases, the loss of graft function sometimes means patient death. The high late mortality rate to infections (Table 2 Part a) can be partially explained by the absence of vaccinations in our patients. Despite ESRD, intensive vaccination is not an established rule in our country. We believe that pretransplant vaccination against meningococ- cal, pneumococcal, and hemophilus infections could have saved lives in patients $3,6,7$, and 8. Aggressive pre- and post-transplant vaccinations have been widely used since 2010 , and we expect a positive change in patient survival rates. Our protocol allowed us to achieve nondeath-censored graft survival rates comparable to those observed by NAPRTCS, despite infection-induced mortality.

The majority of patients received MMF and a CNI, with early discontinuation of steroids. The incidence of biopsy-proven rejection (including borderline and subclinical) at one, two, and three yr was $26 \%, 35 \%$, and $35 \%$, respectively. The incidence of clinical Banff 1a or worse rejection at one, two, and three yr was twice less $13 \%, 18 \%$, and $18 \%$. Rejection was less frequent in tacrolimus-treated patients; overall, three-yr incidence was 19\% for all rejections (including borderline and subclinical) and $10 \%$ for clinical Banff 1a or worse rejections. We would like to be careful with conclusions when comparing two 
Kaabak et al.
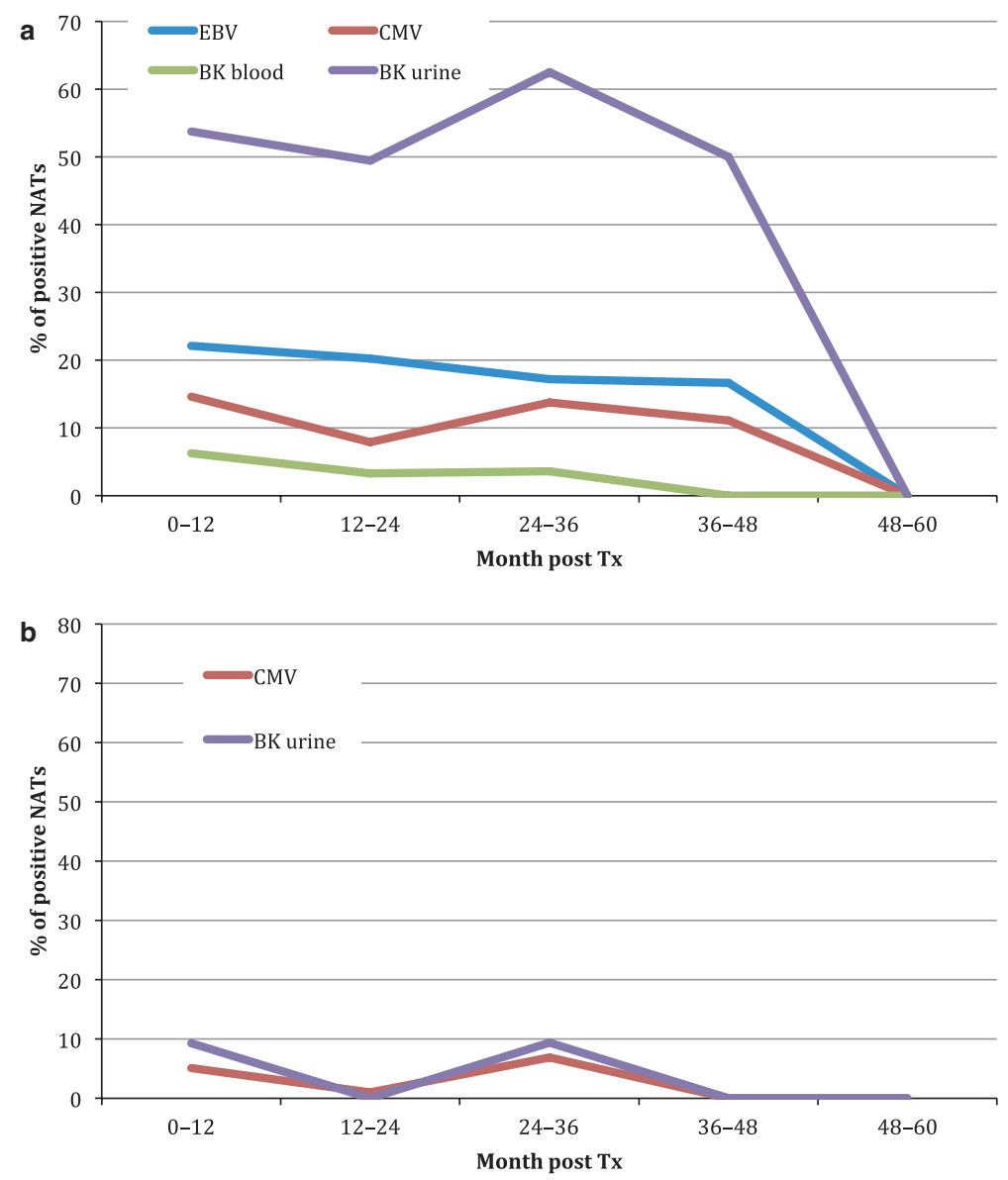

\begin{tabular}{|l|l|l|l|l|l|}
\hline $\begin{array}{c}\text { number } \\
\text { of NUTs }\end{array}$ & 871 & 379 & 118 & 72 & 4 \\
\hline
\end{tabular}

Fig. 6. (a) CNI-free patients at the time of sampling. Positive nucleic acid tests regardless of viral load, as a function of time without CNI. (b) CNI-free patients at the time of sampling. $\mathrm{BK}$ viruria more than $10^{7}$ copies $/ \mathrm{mL}$ and CMV of $10^{3}$ or more copies $/ \mathrm{mL}$ - generally accepted as "dangerous" viral loads - considered as positve.
CNIs in this article, because majority of patients are not randomized and duration of follow-up in tacrolimus group is shorter.

The term "rejection" may have a different meaning in different studies. Thus, the most cited publication on kidney transplantation morphology, describing long-term follow-up of 120 patients, reported rejection rate of $71.4 \%$. However, these rejections had only a limited effect on grafts, because the 10-yr death-censored kidney graft survival rate was $95.2 \%$ (20). This controversy, as well as a high discrepancy in reported rejection rates, can be explained by different definitions of rejection. In our patients, the criteria for rejection were any morphological signs according to Banff classification $(21,22)$, regardless of graft function.

There were six patients with FSGS and nine patients with HUS in our cohort. Among the FSGS patients, no recurrence was seen; two adolescent girls lost a graft to noncompliance: one developed acute rejection eight months posttransplant, refused steroid treatment, and lost the graft seven months later; the second suffered depression 14 months post-transplant after the death of her grandmother, refused oral intake of food, water, and medications, and lost the graft due to rejection. In the HUS patients, one threeyr-old boy had an early recurrence with acute renal failure that was partially resolved after six months, and subsequent graft function was maintained for three yr with a GFR of around $30 \mathrm{~mL} / \mathrm{min}$. He then developed a left pulmonary artery thrombosis. The course of ensuing thrombolysis was complicated with graft rupture and abdominal bleeding, and the graft was removed. The remaining eight HUS patients have had a favorable postoperative course. The details of long-term follow-up in FSGS and HUS patients are summarized in Table 3.

The major concern of alemtuzumab induction in pediatric transplantation is safety. The incidence of serious infections was relatively low, with clinically apparent CMV and EBV infections in $2 \%$ and $1 \%$, respectively (Table 2 Part b). However, the subclinical disease detected by rising titers of viral load was detected in higher levels (Fig. 3) and required a modification of 
Table 3. Post-transplant course in FSGS and HUS patients

\begin{tabular}{lll}
\hline & FSGS & HUS \\
\hline Number of patients & 6 & 9 \\
Patients with rejections & 2 & 1 \\
Long-term graft survival & 4 of 6 & 8 of 9 \\
At last control, days post-transplant & $1390 \pm 569$ & $1080 \pm 146$ \\
Graft function & & \\
$\quad$ Serum creatinine, mg\% & $0.7 \pm 0.2$ & $0.8 \pm 0.2$ \\
$\quad$ Proteinuria, mg/24 h & $45 \pm 55$ & $69 \pm 48$ \\
$\quad$ Calculated GFR, Schwartz formula, $\mathrm{mL} / \mathrm{min}$ & $132 \pm 42$ & $101 \pm 26$ \\
Immunosuppression & & \\
$\quad$ PSI + MMF & 2 & 3 \\
$\quad$ Tacrolimus + MMF & 1 & 3 \\
$\quad$ Cyclosporine + MMF & 1 & 1 \\
Pred + CsA + Aza & & 1 \\
\hline
\end{tabular}

immunosuppression. There were no cases of PTLD, possibly because alemtuzumab also affects B cells.

At three yr, $84 \%$ of patients remained steroid free and $26 \%$ of patients were CNI free (Fig. 1). Apart from lower viral load and less nephrotoxicity, CNI-free immunosuppression can result in decreased donor-specific recipient $\mathrm{T}$-cell reactivity and can accelerate the development of recipients' FOXP3+ Tregs in renal transplant recipients, perhaps enhancing the potential for graft acceptance $(23,24)$.

Separate analysis of the outcomes in children who initially received cyclosporine or tacrolimus is summarized in Table 4, Figs. 4 and 5. However, conclusions should be drawn carefully when interpreting the results, because the majority of these patients are not randomized. Thus, three-yr graft survival rates are superior in the tacrolimus group, but the follow-up duration is shorter. The rejection rate was significantly lower in tacrolimus patients. The viral infection incidence is presented as percentage of positive NATs (Figs. 4-6). The EBV infection rate in tacrolimus-treated patients was $27 \%$ between years 2 and 3 (Fig. 4). New-onset diabetes mellitus was seen in two $(5 \%$ and $6 \%)$ tacrolimustreated patients during treatment for rejection with intravenous and oral steroids; in both cases, insulin was used for glucose control. After tapering of oral steroids, insulin dependence diminished, and after six wk in the former case and three months in the latter, the children maintained a normal glucose metabolism without antidiabetic medications.

Obviously, this report has several important limitations. This is a retrospective analysis with no control groups. Prolonged follow-up will be required to assess long-term patient and graft survival and function, as well as the rate of infectious and malignant complications. Prospective
Table 4. Results of kidney transplantation in children under alemtuzumab induction and received initially maintenance immunosuppression based either on cyclosporine or tacrolimus

\begin{tabular}{lcc}
\hline & Cyclosporine & Tacrolimus \\
\hline Number of patients & 63 & 36 \\
Acute rejections, patients (\%) & $27(43)$ & $7(19)$ \\
Follow-up, d & $1390 \pm 506$ & $1112 \pm 305$ \\
Three-yr survival, \% & & \\
$\quad$ Patient & $90 \pm 4$ & $97 \pm 5$ \\
$\quad$ Graft & $83 \pm 7$ & $97 \pm 5$ \\
\hline
\end{tabular}

trials evaluating alemtuzumab-pretreatment protocols with different post-transplant immunosuppressive regimens are warranted.

\section{Acknowledgments}

This work was supported in part by the Health Resources and Services Administration contract 234-2005-370011C. The content is the responsibility of the authors alone and does not necessarily reflect the views or policies of the Department of Health and Human Services, nor does it mention trade names, commercial products, or organizations that imply endorsement by the U.S. Government. The authors are grateful to Sir Roy Calne for his valuable advice during the study and help with the structure of the manuscript and to Ron Shapiro for editing the manuscript draft.

\section{Authors' contributions}

Michael M. Kaabak, participated in study design and research and in writing of the article and conducting of research. Nadezda N. Babenko, participated in study design and research. Dmitry V. Samsonov, participated in research and writing of the article. Valery A. Sandrikov, participated in study design. Alexey A. Maschan, participated in study design. Alan K. Zokoev, participated in data collection, analysis, and interpretation.

\section{References}

1. Flynn JM, Byrd JC. Alemtuzumab monoclonal antibody therapy. Curr Opin Oncol 2000: 12: 574-581.

2. Ratzinger G, Reagan Jl, Heller G, Busam KJ, Young JW. Differential CD52 expression by distinct myeloid dendritic cell subsets: Implications for alemtuzumab activity at the level of antigen presentation in allogeneic graft-host interactions in transplantation. Blood 2003: 101: 1422-1429.

3. Kirk AD, Hale DA, Mannon RB, et al. Results from a human renal allograft tolerance trial evaluating the humanized CD52-specific monoclonal antibody alemtuzumab (CAMPATH-1H). Transplantation 2003: 76: 120-129.

4. Hale G, Dyer MJ, Clark MR, et al. Remission induction in non-Hodgkin lymphoma with reshaped human monoclonal antibody CAMPATH-1H. Lancet 1998: 2: 1394-1399.

5. Kirsch BM, Haidinger M, Zeyda M, et al. Alemtuzumab (Campath-IH) induction therapy and dendritic cells: Impact on peripheral dendritic cell repertoire in renal allograft recipients. Transpl Immunol 2006: 16: 254-257.

6. Calne R, Friend P, Moffatt S, et al. Prope tolerance, perioperative Campath $1 \mathrm{H}$, and low-dose cyclosporine monotherapy in renal allograft recipients. Lancet 1998: 351: 1701.

7. Watson CJE, Bradley JA, Friend PJ, et al. Alemtuzumab (CAMPATH $1 \mathrm{H})$ induction therapy in cadaveric kidney trans- 


\section{Kaabak et al.}

plantation - Efficacy and safety at five years. Am J Transplant 2005: 5: 1347-1353.

8. Tan HP, Donaldson J, Basu A, et al. Two hundred living donor kidney transplantations under alemtuzumab induction and tacrolimus monotherapy: 3-year follow-up. Am J Transplant 2009: 9: 355-366.

9. Hanaway MJ, Woodle ES, Mulgaonkar S, et al. Alemtuzumab induction in renal transplantation. N Engl J Med 2011: 364: 1909-1919.

10. Chan K, Taube D, Roufosse C, et al. Kidney transplantation with minimized maintenance: Alemtuzumab induction with tacrolimus monotherapy - An open label, randomized trial. Transplantation 2011: 92: 774-780.

11. Calne R, Watson CJE. Some observations in prope tolerance. Curr Opin Organ Transplant 2011: 16: 353-358.

12. Bartosh SM, Knechtlen SJ, Sollinger HW. Campath-1H use in pediatric renal transplantation. Am J Transplant 2005: 5: 1569-1573.

13. Shapiro R, Ellis D, Tan HP, et al. Antilymphoid antibody preconditioning and tacrolimus monotherapy for pediatric kidney transplantation. J Pediatr 2006: 148: 813-818.

14. Tan HP, Donaldson J, Ellis D, et al. Pediatric living donor kidney transplantation under alemtuzumab pretreatment and tacrolimus monotherapy: 4-year experience. Transplantation 2008: 86: 1725-1731.

15. Barratt-Boyesa SM, Thomson AW. Dendritic cells: Tools and targets for transplant tolerance. Am J Transplant 2005: 5: 2807-2813.

16. Vincenti F, Larsen C, Durrbach A, et al. Costimulation blockade with belatacept in renal transplantation. N Engl J Med 2005: 353: 770-781.
17. Gaspari F, Anedda MF, Signorini O, et al. Prediction of cyclosporine area under the curve using a three-point sampling strategy after Neoral administration. J Am Soc Nephrol 1997: 8: 647-652.

18. Momynaliev KT, Gorbatenko EV, Shevtsov AB, Gribanov OG, BABENKo NN, KaABAK MM. Prevalence and subtypes of BK virus in pediatric renal transplant recipients in Russia. Pediatr Transplant 2012: 16: 151-159.

19. NAPRTCS. Annual Transplant Report, 2010. Available at: https://web.emmes.com/study/ped/annlrept/2010_Report.pdfh ttps://web.emmes.com/study/ped/annlrept/annlrept.html (accessed April 26, 2012).

20. Nankivell BJ, Borrows RJ, Chir B, et al. The natural history of chronic allograft nephropathy. N Engl J Med 2003: 349: 2326-2333.

21. Racusen LC, Solez K, Colvin RB, et al. The Banff 97 working classification of renal allograft pathology. Kidney Int 1999: 55: 713-723.

22. Solez K, Colvin RB, Racusen LC, et al. Banff 07 classification of renal allograft pathology: Updates and future directions. Am J Transplant 2008: 8: 753-760.

23. Van Der Mast BJ, Rischen-Vos J, De Kuiper P, Vaessen LM, Van Besouw NM, Weimar W. Calcineurin inhibitor withdrawal in stable kidney transplant patients decreases the donor-specific cytotoxic $\mathrm{T}$ lymphocyte precursor frequency. Transplantation 2005: 80: 1220-1225.

24. Van De Wetering J, Koumoutsakos P, Peeters A, et al. Discontinuation of calcineurin inhibitors treatment allows the development of FOXP3+ regulatory T-cells in patients after kidney transplantation. Clin Transplant 2011: 25: 4046. 